\title{
Viral Etiological Factors Causing Acute Encephalitis Syndrome (AES) In Gaya Division, India
}

\author{
Roshan Kamal Topno ${ }^{1 *}$, Krishna Pandey ${ }^{2}$, Banke Bihari Singh ${ }^{3}$, Manas Ranjan Dikhit ${ }^{4}$, Ashish Kumar ${ }^{5}$, \\ Maneesh Kumar ${ }^{6}$, Ganesh Chandra Sahoo ${ }^{4}$, Vidya Nand Rabidas ${ }^{2}$, Niyamat Ali Siddiquii ${ }^{7}$ Wakil Paswan ${ }^{3}$, \\ Arjun Lal ${ }^{6}$, Diwakar Singh Dinesh ${ }^{9}$, Pradeep Das ${ }^{10^{*}}$
}

\begin{abstract}
'Department of Epidemiology, ICMR-Rajendra Memorial Research Institute of Medical Sciences, Agamkuan, Patna-800007, India ${ }^{2}$ Department of Clinical Medicine, ICMR-Rajendra Memorial Research Institute of Medical Sciences, Agamkuan, Patna-800007, India

${ }^{3}$ Department of Pediatrics, Anugrah Narayan Magadh Memorial Medical College Hospital, Gaya- 823001, India

${ }^{4}$ Department of Bioinformatics, ICMR-Rajendra Memorial Research Institute of Medical Sciences, Agamkuan, Patna-800007, India

${ }^{5}$ Department of Biochemistry, ICMR-Rajendra Memorial Research Institute of Medical Sciences, Agamkuan, Patna-800007, India ${ }^{6}$ Department of Virology, ICMR-Rajendra Memorial Research Institute of Medical Sciences, Agamkuan, Patna-800007, India ${ }^{7}$ Department of Bio-Statistics, ICMR-Rajendra Memorial Research Institute of Medical Sciences, Agamkuan, Patna-800007, India ${ }^{8}$ Department of Microbiology, Anugrah Narayan Magadh Memorial Medical College Hospital, Gaya-823001, India

${ }^{9}$ Department of Vector Biology and Control, ICMR- Rajendra Memorial Research Institute of Medical Sciences, Agamkuan, Patna-800007, India

${ }^{10}$ Department of Molecular Biology, ICMR-Rajendra Memorial Research Institute of Medical Sciences, Agamkuan, Patna-800007, India
\end{abstract}

*Corresponding Authors: 1. Roshan Kamal Topno, Department of Epidemiology, ICMR-Rajendra Memorial Research Institute of Medical Sciences, Agamkuan, Patna- 800007, Bihar, India. Tel: +91-9162501901, Email: roshanktopno@yahoo.co.in

2. Pradeep Das, Department of Molecular Biology, ICMR-Rajendra Memorial Research Institute of Medical Sciences, Agamkuan, Patna-800007, India.Tel:+91-9431012380,Email:drpradeep.das@gmail.com

Received May 11, 2019; Accepted September 5, 2019; Online Published September 15, 2019

\begin{abstract}
Background: From Gaya and adjoining regions, the trend in patients admitted with acute neurological illness was investigated. IIInesses were identified as sudden outbreaks of Japanese virus encephalitis (JE), Herpes simplex virus encephalitis (HSV$1 \& 2$ ), and other acute encephalitis syndrome (AES).

Objective: In the current study, an investigation was carried out to assess potential infectious pathogens in patients aged 16 years or younger who were admitted to Anugrah Narayan Magadh Memorial Medical College Hospital, Gaya, with encephalitis-like symptoms.

Methods: Cross-epidemiological, serological, and molecular biological studies were performed on samples collected from 71 patients below 16 years of age. Patients' clinical histories, i.e. fever, socio-demographic characteristics, and other clinical data, were extracted from patient files.

Results: The results showed confirmed AES cases, including $49.30 \%$ JE and $7.04 \%$ HSV positive patients. A higher casefatality rate of $40 \%$ in JE and $40 \%$ HSV cases below 7 years of age were observed during treatment would become an unavoidable concern. The epidemical sex ratio was observed to be higher in girls than in boys (1.26:1).

Conclusion: The results suggested that JE virus was found to be a main causative risk factor responsible for disease transmission in the outbreak area.

Keywords: Acute Encephalitis Syndrome, Enzyme-Linked Immunosorbent Assay (ELISA), Herpes Simplex Virus (HSV), Japanese Encephalitis, Outbreak
\end{abstract}

\section{Background}

Acute encephalitis syndrome (AES) is a multifaceted clinical health syndrome often associated with substantial illness along with high morbidity and mortality. The syndrome is well defined as the acute arrival of illness that eventually occurs as seasonal outbreak. ${ }^{1}$ The severe disease causes inflammation in the brain and leads to other neurological conditions like convulsions, autonomic disturbance, abnormal behavior, episodic headache, mood disorder, confusion, disorientation, delirium, or even

Copyright (C 2019 The Author(s). This is an open-access article distributed under the terms of the Creative Commons Attribution License (http:// creativecommons.org/licenses/by/4.0), which permits unrestricted use, distribution, and reproduction in any medium, provided the original work is properly cited. 
coma. It emerged as a major public health problem in Asian countries. $^{2}$ This dreadful condition, thought to be caused by a wide variety of pathogens including bacterial or viral infections in the brain, ingestion of toxic substances, has been reported in the past few decades. These etiological agents usually vary with geographical regions, ${ }^{3,4}$ and they lead to more complicated infections with severe underlying malignancy.

More than 100 diverse pathogens have been identified as causative agents for AES. Among them, Japanese virus encephalitis (JE), Herpes simplex virus encephalitis (HSV, varicella-zoster, the Epstein-Barr virus, mumps, measles, enteroviruses, influenza, adenovirus, echovirus, and mycoplasma pneumonia are the most frequent pathogens. ${ }^{5,6}$ $\mathrm{JE}$ is a vector-borne viral infirmity that accounts for nearly $15 \%$ of AES cases, and the etiology of AES in $68 \%-75 \%$ of cases remains unknown. Between January 2008 and August 2014, nearly 44097 cases with 5728 deaths due to AES were reported in India. ${ }^{7,8}$ This number represents a considerable increase in the annual number of reported cases. Among these, Japanese encephalitis is considered one of the major causes of AES in India. ${ }^{9}$ More specifically, the areas most afflicted by the AES outbreak are the eastern part of Uttar Pradesh and the districts of Muzaffarpur and Gaya from Bihar. ${ }^{10}$ However, no large scale study has investigated the specificity of causative agents of AES in Gaya and its adjoining regions.

\section{Objective}

AES excessively affects children below the age of 16 years and poses a severe risk to the lives of affected children. Most underlying illnesses are hardly ever investigated in order to understand the possible agent and its means of spread. Recently, in India, an outbreak of AES led to the deaths of several children in Bihar. These cases were well characterized by sudden onset of fever, occasional neck rigidity, restlessness, convulsion leading to coma, followed by death within 9-10 days of acute illness onset. During or after the rainy season from June to October, JE is more prevalent. ${ }^{11-14}$ It is therefore very crucial that detailed epidemiological investigations be conducted that will explore the hidden factors involved in this dreadful disease.

\section{Methods}

From June to October 2016, cerebrospinal fluid (CSF) and serum samples were collected from 71 patients of different age groups to check for clinically suspected viral encephalitis in Anugrah Narayan Magadh Memorial Medical College Hospital, Gaya, India. People residing in the area of the hospital are from a low socio-economic group. Male and female patients aged between 0.3-16 years were included in the study (Notably, all cases in this study were below 16 years of age), and their clinical history and symptoms were documented. CSF was collected from patients with suspected cases of encephalitis and without contraindications to lumbar puncher, according to the judgment of the responsible pediatrician. Samples were sent to ICMR-Rajendra Memorial Research Institute of Medical Sciences (RMRIMS), Patna, India, and stored at $-80^{\circ} \mathrm{C}$. The current study was carried out in Virology Lab, RMRIMS, Patna.

CSF/serum samples of 71 clinically positive cases were investigated for JE, Leptospira, chikungunya, HSV-1\&2, measles, dengue, or scrub typhus specific IgM antibodies using an enzyme-linked immunosorbent assay (ELISA) kit (Calbiotech, USA). Briefly, the wells of microtiter plates were coated with $75 \mathrm{~mL}$ of anti-human immunoglobulin $\mathrm{M}$ ( $\operatorname{IgM})$, followed by the addition of $50 \mathrm{~mL}$ of serum (1:100 dilution) or CSF (1:10 dilution), biotinylated antiflavivirus antibody, avidin-horseradish peroxidase (HRP) conjugate, and substrate. Calculations were performed per the manufacturer's instructions after taking the optical density (OD) values at $492 \mathrm{~nm}$. Moreover, CT scans were performed specifically on HSV-positive samples.

RNA genome isolation (using QIAamp Viral RNA Mini Kit, QIAGEN, USA) and real-time-polymerase chain reaction (RT-PCR) (using one-step RT-PCR kit, Takara) for Japanese encephalitis virus (JEV) were carried out on all blood and CSF samples from patients following the method described by Paranjpe et al, with minor modifications where required. ${ }^{13,14}$ Briefly, ribonucleic acid (RNA) was extracted from $200 \mu \mathrm{L}$ of serum/CSF sample using TRIzol reagent (Invitrogen). Reverse transcription was performed using an RT kit (Invitrogen). PCR was carried out using the appropriate primer pair as stated (Table 1). In each PCR reaction, the appropriate positive and negative controls were included for sample confirmation. The sensitivity of the PCR was determined with complementary DNA (cDNA) made by reverse transcription of JEV RNA from the reference strain. Amplified DNA fragments were visualized through ethidium bromide staining following $2 \%$ agarose gel electrophoresis. JE specific IgM or RT-PCR positivity in the CSF samples were taken as the criteria for confirming Japanese encephalitis cases. Similarly, PCR was also carried out for the presence of the West Nile virus.

\section{Results}

Gaya is located 62 miles south of Patna, the capital city of Bihar (longitude: 84.4 to 85.5 degrees toward east; latitude: 24.5 to 25.10 degrees toward north) and has a humid subtropical climate (Köppen climate classification). Relatively high temperate weather and evenly distributed precipitation were observed throughout the year. The environmental circumstances are very favorable for

Table 1. The Pprimer Used to Detect the Presence of Either Japanese Encephalitis or West Nile Virus in Clinically Suspected Cases

\begin{tabular}{llc}
\hline Species & Primers (Forward and Reverse) & Product Size \\
\hline $\begin{array}{l}\text { Japanese } \\
\text { encephalitis }\end{array}$ & F:- GGAAGGCCTGGGGGCAGGACGCTA & 460 \\
$\begin{array}{l}\text { West Nile } \\
\text { virus }\end{array}$ & F:- GCTCCGCACCCTGTCTTCCTGGATCAA & \\
\hline
\end{tabular}


mosquitoes breeding. Although little information is available about the periodic outbreaks of JEV, a local newspaper confirms the spread and death due to AES. In this study, an in-depth investigation was carried out to explore the exact scenario of the JE outbreak in the Gaya district of Bihar to support public health measures. Due to low levels of viremia and the fast development of neutralizing antibodies, RT-PCR is slightly tricky and depends mostly on the time blood/CSF specimens are taken. ${ }^{15-17}$ Although controversial, the detection rate is often more accurate than that of IgM-capture ELISA for serum and CSF. ${ }^{18,19}$ Therefore, both IgM-capture ELISA and RT-PCR were performed for the diagnosis of suspected cases from Gaya, Bihar.

A total of 71 clinically suspected cases less than 16 years of age were included in the current study. Almost all cases came from Gaya or its adjoining areas. The presenting symptoms in these suspected cases were high-grade fever, headache, vomiting, lethargy, altered sensorium, and convulsion. The 71 positively-confirmed AES cases were included for further study. Blood and CSF specimens were taken during June-October 2016.

The data obtained from ELISA and RT-PCR revealed at least $49.30 \%$ (35/71) of patients were clinically confirmed JEV cases. Nearly $37.14 \%$ (13/35) and 40\% (14/35) of children between the ages of 0-4 and 5-9 years, respectively, were affected. Fascinatingly, $22.86 \%$ (8/35) children of the $10-16$ years age group were badly affected by this deadly virus. Though several studies indicated a soaring rate of boys infected as compared to girls, the current study conceded a higher rate of girls affected. Interestingly, the epidemic affected girls and boys at a ratio of 1.26:1. More importantly, this ratio is quite high (1.7:1) among children below the age of 9 years. Samples were collected randomly, and the girl:boy ratio of suspected JE sample was 1.24:1. Even though there may be several epidemiological or other vital reasons, the altered sample collection may partially affect the infected girl:boy ratio. A detailed epidemiological investigation could explore the reasons why girls are more prone to disease than boys. The overall case-fatality rate was $40 \%$ (14/35), of which $60 \%$ were girls and $40 \%$ were boys. Around $90 \%$ of the fatality cases were below 9 years of age.

\section{Discussion}

Out of the total number of suspected JEV samples, 35 were found to be IgM positive. Both ELISA and PCR were carried out for the diagnosis of JE-positive cases (Figures $1 \mathrm{~A}$ and $\mathrm{B})$. As previously reported, both the sensitivity and specificity of ELISA are more than 95\% after the 7th day of disease onset, and specimens collected before 7 days may be negative. ${ }^{20}$ Due to a high degree of sensitivity and specificity, the RT-PCR method has a potential application for routine diagnosis of flaviviral infections. More importantly, the samples collected from the areas where JE and dengue are both endemic may show cross-reactivity at the IgM level. ${ }^{21}$ Therefore, both IgM-negative and -positive
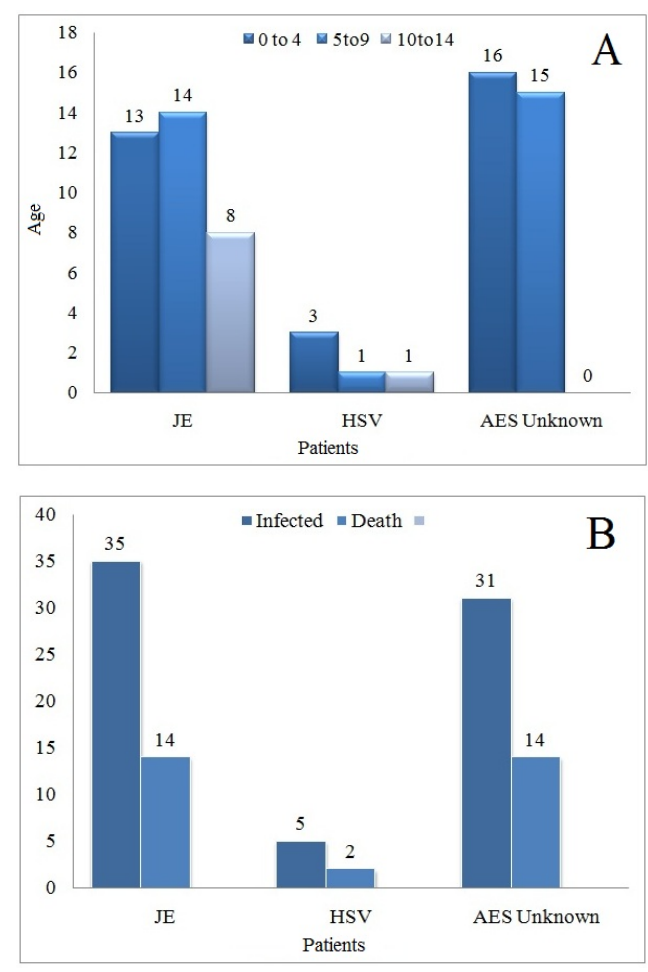

Figure 1. Age group distribution of JE, HSV, and AES due to unknown pathogens and their fatality rates are mentioned in graphical representation.

samples were especially investigated for RT-PCR analysis. Specifically, 4 samples were found to be positive by RT-PCR alone, whereas 14 samples were found to be positive by both ELISA and RT-PCR. This information indicated that the samples in the current study were probably collected within the first 7 days of illness when the antibody titer was moderate in the presence of viral RNA. More precisely, $11.42 \%$ of the samples, those found to be positive by RTPCR (Figure 2B), reflected only the possibility of a high viral load with a scanty level of neutralizing antibodies. Due to the short toxemia period of JE patients, only 18 RTPCR-positive (51.43\%) JEV-infected cases were detected using the RT-PCR approach.

\section{Conclusion}

The total number of confirmed AES cases that occurred during the epidemic was 71 . The meticulous analysis performed in this study revealed that nearly $49.30 \%$ of encephalitis cases occurred due to JEV infection (Figure 2A). To search for other factors responsible for AES in Gaya and its adjoining regions, the samples were tested for HSV$1 \& 2$ (IgM) and scrub typhus (IgM). Previously, Murhekar et al reported that scrub typhus (63\% of AES cases) was one of the major etiologies for acute encephalitis. In contrast to these findings, the current data suggested that the AES cases near Gaya and its adjoining regions were negative to scrub typhus. ${ }^{22,23}$ Interestingly, HSV-1\&2 was found to be a second causal factor for AES confirmed by both ELISA (Figure 2A) and contrast-enhanced computed tomography (CECT) scan (Figure 2C). More importantly, 5 HSV cases 


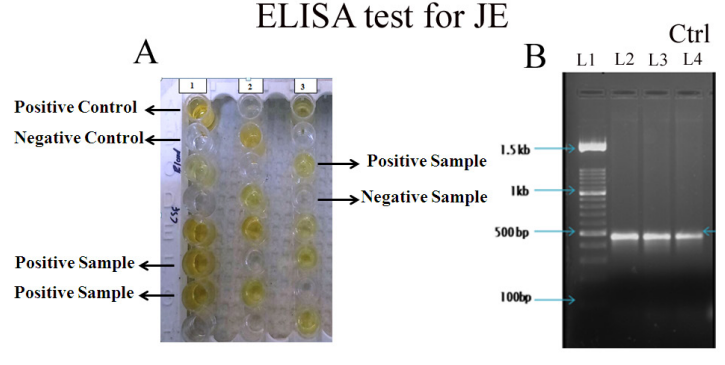

C

CECT brain images of HSV cases

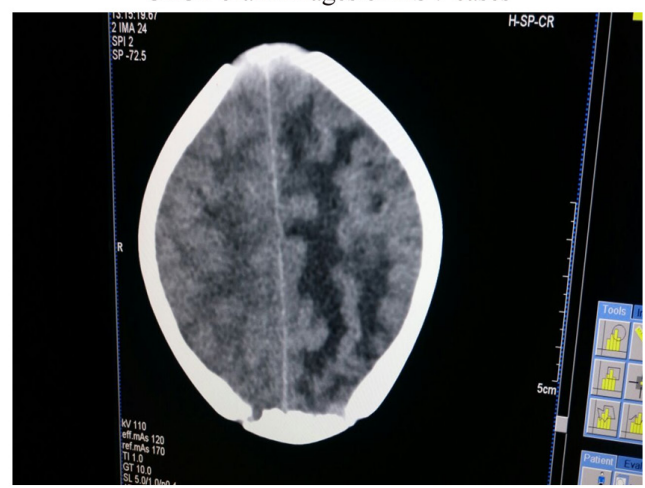

Figure 2. Representative Figures Showing (A) JE-Positive Samples by ELISA; (B) JE-Positive Samples by PCR; and (C) HSV-Positive Samples by CECT Brain Scan.

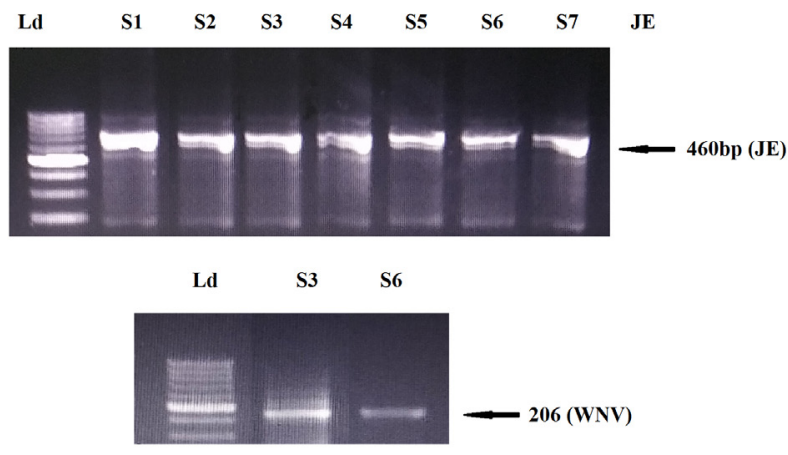

Figure 3. PCR Confirmation of JE and WNV

(7.04\%) were detected with an overall fatality rate of $40 \%$ $(2 / 5)$. The further confirmation of JE and WNV infections were confirmed by performing qPCR (Figure 3).

Among the 71 AES cases, $35(49.30 \%)$ cases and 5 (7.04\%) cases were found to be JE- and HSV-positive, respectively. The foremost result of this study revealed that all confirmed AES cases were under the age of 9 years. As the landscape of AES, outbreaks have altered in the past few years, and both outbreak investigations and surveillance studies have increasingly reported non-JEV etiologies. The deliberate investigation conducted in this study revealed that none of the AES-confirmed cases were positive to other known viruses causing acute encephalitis. The main causative agent(s) remain unexplained..$^{24}$ It can now be concluded that JEV is the main etiological agent responsible for AES. However, a greater number of deaths occurred due to unrecognized etiological agents that need
Research Highlights

\section{What Is Already Known?}

Acute encephalitis syndrome (AES) has been a major health problem in Gaya, Bihar with high morbidity and mortality.

\section{What This Study Adds?}

The result suggested that JE virus followed by HSV were the causative agents for viral encephalitis.

to be identified. A detailed investigation is required to determine the unknown causative agents responsible for AES near Gaya and its adjoining regions.

\section{Authors' Contributions}

RKT, KP, BBS, AL, PD contributed to the study design, conceptualization, and data collection; MRD, NAS, MK, GCS, PD contributed to data interpretation and manuscript preparation; VNRD, WP, DSD, AK, PD participated in manuscript revision; RKT contributed to manuscript submission.

\section{Conflict of Interest Disclosures}

The authors affirm that they have no conflicts of interest.

\section{Ethical Approval}

The current study was approved by the Institutional Ethical Committee, ICMR-Rajendra Memorial Research Institute of Medical Sciences, Agam-kuan, Patna, India.

\section{References}

1. Narain JP, Dhariwal AC, Maclntyre CR. Acute encephalitis in India: An unfolding tragedy. Indian J Med Res. 2017;145(5):584587. doi:10.4103/ijmr.IJMR_409_17.

2. Sen PK, Dhariwal AC, Jaiswal RK, Lal S, Raina VK, Rastogi A. Epidemiology of acute encephalitis syndrome in India: changing paradigm and implication for control. J Commun Dis. 2014;46(1):4-11.

3. Granerod J, Crowcroft NS. The epidemiology of acute encephalitis. Neuropsychol Rehabil. 2007;17(4-5):406-428. doi:10.1080/09602010600989620.

4. Le VT, Phan TQ, Do QH, et al. Viral etiology of encephalitis in children in southern Vietnam: results of a one-year prospective descriptive study. PLoS Negl Trop Dis. 2010;4(10):e854. doi:10.1371/journal.pntd.0000854.

5. Borkotoki $U$, Borkotoki S, Barua $P$, et al. Japanese encephalitis (JE) among acute encephalitis syndrome (AES) cases-a hospital based study from upper Assam, India. Int J Health Sci Res. 2016;3:4-10. 6. Goel S, Chakravarti A, Mantan M, Kumar S, Ashraf MA. Diagnostic approach to viral acute encephalitis syndrome (AES) in paediatric age group: a study from New Delhi. J Clin Diagn Res. 2017;11(9):DC25-DC29. doi:10.7860/ jcdr/2017/27413.10648.

6. Nayak P, Papanna M, Shrivastava A, et al. Unexplained neurological illness in children, Malkangiri district, Odisha, India 2014. International Journal of Infectious Diseases. 2016;45:305. doi:10.1016/j.ijid.2016.02.668.

7. Kumar P, Pisudde PM, Sarthi PP, Sharma MP, Keshri VR. Status and trend of acute encephalitis syndrome and Japanese 
encephalitis in Bihar, India. Natl Med J India. 2017;30(6):317320. doi:10.4103/0970-258x.239070.

8. Ghosh S, Basu A. Acute Encephalitis Syndrome in India: The Changing Scenario. Ann Neurosci. 2016;23(3):131-133. doi:10.1159/000449177.

9. Singh GK, Singh CM, Ranjan A, et al. Determinants of Acute Encephalitis Syndrome (AES) in Muzaffarpur district of Bihar, India: A case-control study. Clin Epidemiol Glob Health. 2016;4(4):181-187. doi:10.1016/j.cegh.2016.05.002.

10. Kumar A, Kumar R, Kaur J. Japanese encephalitis: medical emergency in India. Asian J Pharm Clin Res. 2012;5(3):9-12.

11. Jacobson J, Sivalenka S. Japanese encephalitis globally and in India. Indian J Public Health. 2004;48(2):49-56.

12. Kumar M, Topno RK, Dikhit MR, et al. Molecular docking studies of chloroquine and its derivatives against P23(pro-zbd) domain of chikungunya virus: Implication in designing of novel therapeutic strategies. J Cell Biochem. 2019;120(10):1829818308. doi:10.1002/jcb.29139.

13. Yang DK, Kim HH, Jo HY, Choi SS, Cho IS. Establishment of a multiplex RT-PCR for the sensitive and differential detection of Japanese encephalitis virus genotype 1 and 3. J Bacteriol Virol. 2016;46(4):231-238. doi:10.4167/jbv.2016.46.4.231.

14. Mei L, Wu P, Ye J, et al. Development and application of an antigen capture ELISA assay for diagnosis of Japanese encephalitis virus in swine, human and mosquito. Virol J. 2012;9:4. doi:10.1186/1743-422x-9-4.

15. Kumar M, Topno RK, Madhukar M, et al. Acute encephalitis syndrome child patient with multi-viral co-infection: A rare case report. J Med Allied Sci. 2019;9(2).

16. Parida M, Dash PK, Tripathi NK, et al. Japanese Encephalitis Outbreak, India, 2005. Emerg Infect Dis. 2006;12(9):14271430. doi:10.3201/eid1209.060200.
17. Karthikeyan A, Shanmuganathan S, Pavulraj S, et al. Japanese encephalitis, recent perspectives on virus genome, transmission, epidemiology, diagnosis and prophylactic interventions. Journal of Experimental Biology and Agricultural Sciences. 2017;5(6):730-748. doi:10.18006/2017.5(6).730.748

18. Verma RK, Singh DP, Yadav R, Rawat R. Comparative evaluation of antigen detection ELISA and reverse transcriptase PCR in acute stage of Japanese encephalitis prevalent in endemic areas of North-Eastern part of Uttar Pradesh, India. Int J Res Med Sci. 2015;3(11):3217-3223. doi:10.18203/2320-6012. ijrms20151166.

19. Robinson JS, Featherstone D, Vasanthapuram R, et al. Evaluation of three commercially available Japanese encephalitis virus IgM enzyme-linked immunosorbent assays. Am J Trop Med Hyg. 2010;83(5):1146-1155. doi:10.4269/ajtmh.2010.10-0212.

20. Puri B, Henchal EA, Burans J, et al. A rapid method for detection and identification of flaviviruses by polymerase chain reaction and nucleic acid hybridization. Arch Virol. 1994;134(1-2):2937. doi:10.1007/bf01379104.

21. Murhekar MV, Mittal M, Prakash JA, et al. Acute encephalitis syndrome in Gorakhpur, Uttar Pradesh, India - Role of scrub typhus. J Infect. 2016;73(6):623-626. doi:10.1016/j. jinf.2016.08.014.

22. Varghese GM, Rajagopal VM, Trowbridge P, Purushothaman D, Martin SJ. Kinetics of IgM and IgG antibodies after scrub typhus infection and the clinical implications. Int J Infect Dis. 2018;71:53-55. doi:10.1016/j.ijid.2018.03.018.

23. Mittal M, Bondre V, Murhekar M, et al. Acute Encephalitis Syndrome in Gorakhpur, Uttar Pradesh, 2016: Clinical and Laboratory Findings. Pediatr Infect Dis J. 2018;37(11):11011106. doi:10.1097/inf.0000000000002099. 